\title{
Atmospheric influence on image quality of airborne photographs
}

Fang Lei*

H. J. TIzianl, FELLOW SPIE

University of Stuttgart

Institute of Applied Optics

Pfattenwaldring 9

0.70569 Stuttgart, Germany

\begin{abstract}
The image quality of airborne photographs is influenced by the atmosphere. Several factors affect the atmosphere, such as temperature, pressure, humidity, and wind speed. Furthermore, these atmospheric influences are dependent on the flight altitude. The MTF of the atmospheric influences and its dependence on the altitude are examined theoretically and experimentally. Several airbome photographs taken at different altitudes have been evaluated with edge gradient analysis as well as with a grating pattern method. Results are shown, discussed, and compared with model calculations.
\end{abstract}

Subject terms: atmospheric imaging; image quality; modulation transfer function; aerial photography.

Optical Engineering 32(9), 2271-2280 (September 1993).

\section{Introduction}

The atmosphere plays an important role in image quality in the image formation in aerial photography. Therefore, it is a nonnegligible link of the image transfer chain. In aerial cameras not yet equipped with a so-called forward motion compensation system (FMC), the image motion is a dominant element for the reduction of the image quality of airborne photographs, ${ }^{1-4}$ and the influence of the atmosphere is very often not taken into consideration. After the compensation of image motion with FMC, the influences of vibration and the atmosphere on the image quality become increasingly important. The atmospheric influence includes, among other things, turbulence, contrast degradation due to atmospheric background, and forward scattering by airborne particles, ${ }^{5-7}$ etc. The atmospheric influences are based on the molecular absorption of gas dissolved in air, on the scattering by aerosol and dust particles, and on local short and long time changes of the refractive index. Besides the visible disturbances, the meteorological parameters still are of great importance, such as atmospheric pressure, humidity, temperature, temperature gradient, and wind speed. These parameters vary with time as well as spatially. Therefore, the problems, which appear in an investigation of the atmosphere, are very complicated and, hence, must be examined with statistical methods. For the investigation of atmospheric influences in optical astronomy some theoretical work was carried out ${ }^{8-11}$ and verified through practical measurements, ${ }^{12}$ whereas the influence of the atmosphere on airborne photographs was often not considered and very few reports on investigations were found..$^{5-7}$

"Present address: Thomson Consumer Electronics, R\&D Laboratories, Villingen, P.O. Box 1307. D-78003 VS-Villingen, Germany.

\footnotetext{
Paper 2990 received Oct. 4, 1990; revised manuscript received Jan. 28, 1992;

o 1993 Society of Photo-O 24, 1993.
}

$0091-3286 / 93 / 56.00$.
In this paper, we study the influences of the atmosphere on image quality of airborne photographs. Different models of the atmospheric MTF are summarized and compared. Results obtained from airborne photographs taken at different altitudes are discussed and compared with theoretical models.

\section{Models to Obtain the Atmospheric MTF}

As illustrated in Fig. 1, the following atmospheric phenomena need to be considered in aerial photography: (1) atmospheric turbulence, (2) contrast degradation due to atmospheric background, 5,6 (3) forward scattering by airborne particles, ${ }^{6}$ and (4) aircraft boundary layer turbulence. ${ }^{7}$ The influence of each phenomenon can be described by an MTF. Figure 1 shows the image transfer chain linked by the different atmospheric MTFs.

\subsection{Atmospheric Turbulence}

The atmospheric turbulence arises from the permanent mixture of cold and warm layers of air, which leads to local density and humidity variations. The density and humidity variations act as temporal and spatial refractive index variations. Intensity variations due to turbulence can be represented approximately by a log-normal distribution. 8.10 The MTF of atmospheric turbulence has been derived from the log-normal model. Using the results of Fried, ${ }^{10}$ it is given by

$\mathrm{MTF}_{T}=\exp \left\{-3.44\left(\lambda f^{\prime} R / r_{0}\right)^{5 / 3}\left[1-\alpha\left(\lambda f^{\prime} R / D\right)^{1 / 3}\right]\right\}$,

where

$D=$ the entrance pupil diameter of the camera

$f^{\prime}=$ the focal length of the objective

$\lambda=$ the wavelength

$R=$ the spatial frequency 


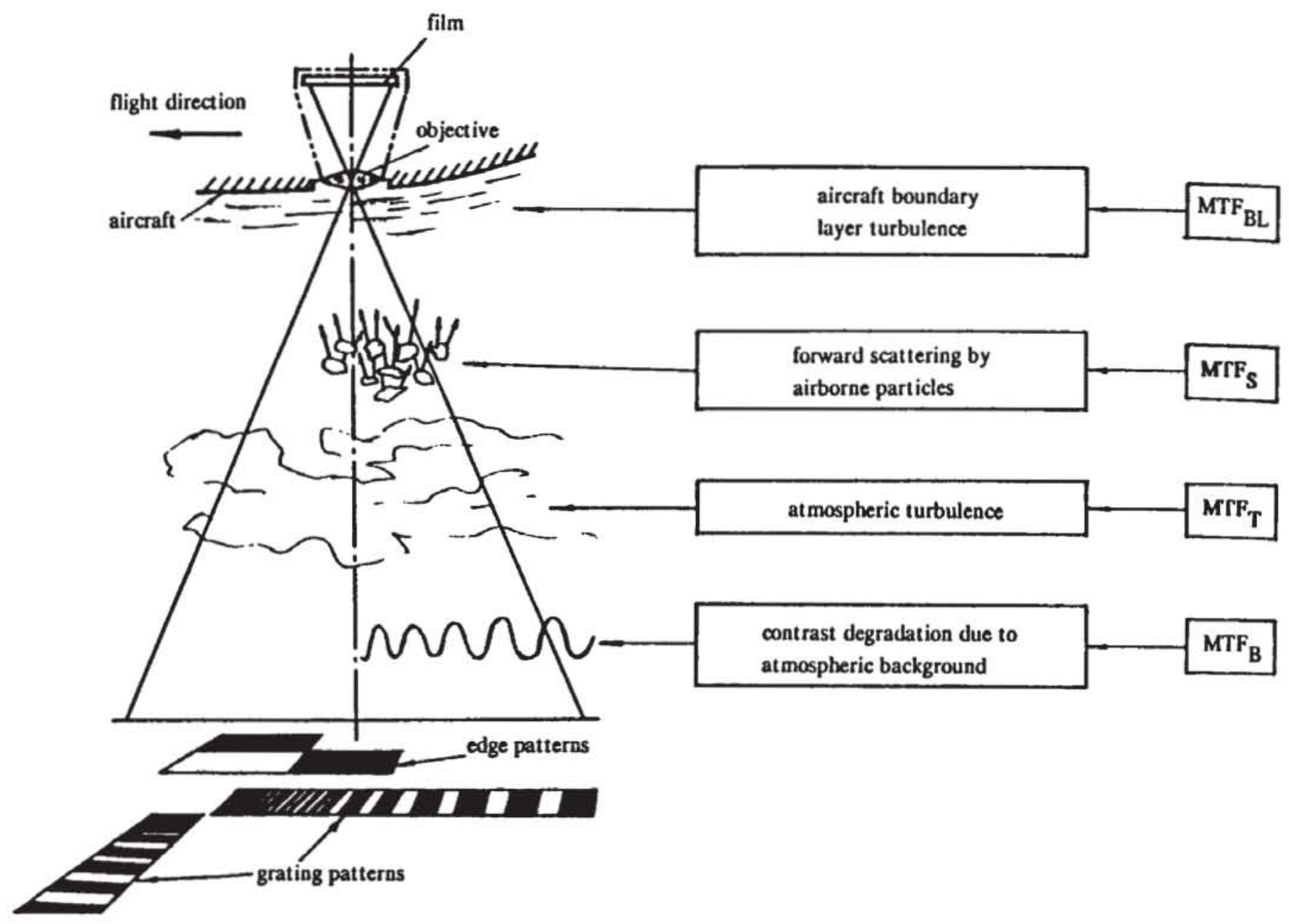

Fig. 1 Schematic representation of different atmospheric influences on image quality of airborne photographs.

$r_{0}=$ Fried's parameter and represents the coherence parameter of the atmospheric wavefront distortions

$\alpha=$ parameter: $\alpha=0$ for the long exposure, $\alpha=1$ for the short exposure in the near-field case, $\alpha=1 / 2$ for the short exposure in the far-field case; $D \gg \sqrt{L \lambda}$ (near field) and $D<<\sqrt{L \lambda}$ (far field); $L$ is the length of the propagation path through the turbulent medium.

Thus, it has been attempted to describe atmospheric turbulence with a single parameter $r_{0}$, which is defined by Fried ${ }^{9}$ as follows:

$r_{0}=0.185\left[\lambda^{-2} \sec \theta \int_{h_{0}}^{H} C_{n}^{2}(h) \cdot \mathrm{d} h\right]^{-3 / 5}$,

where

$\theta=$ the zenith angle

$h_{0}=$ the ground altitude above sea level

$H=$ the flight altitude above sea level

$h=$ variable of the altitude

$C_{n}^{2}=$ the refractive index structure constant.
Typical values of Fried's parameter $r_{0}$ for astronomical observations in the visible spectral range are between 20 and $200 \mathrm{~mm}$. "The mean value of $r_{0}$ has been investigated by Fried and Mevers. ${ }^{13}$ For $\lambda=0.55 \mu \mathrm{m}$ and zenith propagation, $r_{0}=114 \mathrm{~mm}$ with a standard deviation of 1.36 has been derived from analysis of astronomical sight measurements at night at a good observatory sight.

In aerial photography, only short exposures $(T \ll 0.04 \mathrm{~s})$ are usually considered. Thus, $\alpha$ in Eq. (1) cannot be zero. In comparison with a typical observation time in astronomy, the exposure time here is so short that the atmospheric turbulence can be regarded as frozen during the exposure time. Therefore, the entrance angle variation of the wavefront can be neglected in the short exposure while it leads to a stochastic image motion in the long exposure. Short exposure can reduce the influence of atmospheric turbulence.

Astronomical photography attempts to compensate the wavefront distortion with adaptive optics. ${ }^{14}$ The adaptive optical system can control the entrance wavefront in real time and can compensate its deviation by adequate changes of the shape of an active optical component. This is very difficult to realize in aerial photography. Using the methods of digital image processing, a correction of the influences of turbulence on airborne photographs can only take place after the film has been developed. ${ }^{15,16}$ Therefore, the knowledge of atmospheric properties is very important. 


\subsection{Contrast Degradation Due to Atmospheric Background}

Photons entering the imaging system come not only from the target but also from the surroundings of the target and from the atmospheric background between the target and the imaging system. The light from the atmospheric background causes glare, which leads to an image contrast degradation. ${ }^{5}$ The brighter the atmospheric background, the smaller the percentage of photons received that derive from the target itself. The MTF of the contrast degradation is given by ${ }^{6}$

$$
\begin{aligned}
\operatorname{MTF}_{B}= & {\left[\rho_{t}(\lambda, T)+\rho_{b}(\lambda, T)\right] /\left\{\rho_{r}(\lambda, T)+\rho_{b}(\lambda, T)\right.} \\
& \left.+\left[2 \pi N_{A}(\lambda, T) / M_{s}(\lambda)\right] \exp \left[\sec \theta \int_{h_{0}}^{H} \sigma(h, \lambda) \mathrm{d} h\right]\right\}
\end{aligned}
$$

where

$$
\begin{aligned}
& \rho_{t}=\text { the target reflection coefficient } \\
& \rho_{b}=\text { the target background reflection coefficient } \\
& N_{A}=\text { the atmospheric radiance imaged over the path } \\
& \text { length } h_{0}-H \\
& M_{s}=\text { the solar irradiance } \\
& \sigma=\text { the atmospheric extinction coefficient } \\
& T=\text { the absolute temperature. }
\end{aligned}
$$

The exponential term in Eq. (3) is reciprocal to the atmospheric transmission. For $H<10 \mathrm{~km}$, there is ${ }^{6}$ :

$$
\int_{h_{0}}^{H} \sigma \cdot \mathrm{d} h \cong \sigma_{0} \int_{h_{0}}^{H} \exp (-h / 1.2) \mathrm{d} h,
$$

where $\sigma_{0}$ is the extinction coefficient at sea level. According to Eqs. (3) and (4), the MTF of contrast degradation decreases with increasing flight altitude. Equation (3) shows that the MTF due to contrast degradation is independent of spatial frequency. Therefore, the entire MTF should be reduced equally over all spatial frequencies by the atmospheric background.

\subsection{Forward Scattering by Airborne Particles}

There are particles of different size in the atmosphere, for example, haze (particle diameter 0.01 up to $1 \mu \mathrm{m})$, smoke and dust particles $(0.1$ up to $10 \mu \mathrm{m})$, fog and clouds ( 1 up to $100 \mu \mathrm{m})$, rain $(100$ up to $1000 \mu \mathrm{m})$, and snow $(>1 \mathrm{~mm}){ }^{17}$ They cause scattering of light at different angles relative to the direction of propagation. The light scattering at small angles is called the forward scattering. Forward-scattered light at very small angles may enter the imaging system together with the unscattered light, thus it can affect the intensity distribution of the point spread function. The effect of the forward scattering on image quality depends on wavelength and particle size. The MTF of the forward scattering can be written $a^{6}$ :

$$
\begin{cases}\exp \left\{-\left(\frac{R}{R_{c}}\right)^{2} \sec \theta \int_{h_{0}}^{H}\left[S_{a}(h, \lambda)+A_{a}(h, \lambda)\right] \mathrm{d} h\right\} & R<R_{c} \\ \exp \left\{-\sec \theta \int_{h_{0}}^{H}\left[S_{a}(h, \lambda)+A_{a}(h, \lambda)\right] \mathrm{d} h\right\} & R \geqslant R_{c}\end{cases}
$$

where $S_{a}$ is the scattering coefficient and $A_{a}$ is the atmospheric absorption coefficient. In Eq. (5), $R_{c}$ is a spatial frequency cutoff. The MTF of the forward scattering remains unchanged above $R_{c}$ for a given flight altitude and wavelength as shown in the second part of Eq. (5). It is defined by

$$
R_{c}=\frac{a}{\lambda f^{\prime}},
$$

where $a$ represents the scattering particle radius. For example, we suppose the following:

$$
\begin{aligned}
a & =5 \mu \mathrm{m} \text { (smoke and dust particles) } \\
\lambda & =0.55 \mu \mathrm{m} \\
f^{\prime} & =300 \mathrm{~mm} .
\end{aligned}
$$

Then, it results from Eq. (6):

$R_{c}=0.03 \mathrm{~mm}^{-1}$.

This shows that the cutoff frequency $R_{c}$ is very small in the normal case. Thus, only in a very small frequency range is the MTF of the forward scattering dependent on the spatial frequency. This range with very low spatial frequencies is so small that it can be neglected by the evaluation of airborne photographs. Therefore, the MTF of the forward scattering can be considered independent of the spatial frequency as shown in Eq. (5).

\subsection{Aircraft Boundary Layer Turbulence}

Due to the motion of the aircraft through the ambient air, turbulences are produced in a boundary layer directly in front of the objective of the aerial camera. Further turbulence inside the camera can arise from strong thermal gradients imposed by the air within the camera. These turbulences are called aircraft boundary layer turbulences. ${ }^{7}$ They differ from the normal atmospheric turbulences and additionally deteriorate the image quality. In analogy to the MTF of the atmospheric turbulence, the boundary layer turbulence is given by an exponential function?

$$
\mathrm{MTF}_{B L}=\exp \left[-3.44\left(\lambda f^{\prime} R / r_{b l}\right)^{5 / 3}\right] \text {. }
$$

Instead of $r_{0}$ in Eq. (1), another parameter $r_{b l}$ is introduced in Eq. (7) to characterize the boundary layer turbulence: $r_{b l}$ is a correlation length for the boundary layer. There is a hypothesis that $r_{b l}$ should be proportional to $\lambda^{6 / 5}$. This has been confirmed by some experimental data. ${ }^{7}$ Furthermore, the size of the parameter $r_{b l}$ depends on the flight speed, on the air density around the aircraft, and on the surface form of the aircraft, namely, on the type of the aircraft. At visible wavelengths, the values of 5 to $20 \mathrm{~mm}$ have been measured for several aircraft types. ${ }^{18}$ For a flight altitude of $12 \mathrm{~km}, r_{b l}$ between 8.3 and $14.5 \mathrm{~mm}$ were found by Smith. ${ }^{7}$ These val- 
ues of $r_{b t}$ are smaller than all typical values of Fried's parameter (see Sec. 2.1). Thus, the influence of the MTF of aircraft boundary layer turbulence can be stronger than atmospheric turbulence.

The entire atmospheric MTF can be composed of the MTFs mentioned above. One has to consider how the MTFs of the various atmospheric influences can be connected. For the first three MTFs cited above, the following connection has been suggested by Kopeika ${ }^{6}$ :

$$
\begin{aligned}
\operatorname{MTF}_{A}(R, \lambda) \cong & \operatorname{MTF}_{B}(\lambda) \cdot \operatorname{MTF}_{T}(R, \lambda) \\
& \times\left[\frac{I_{u}}{I_{u}+I_{s}}+\frac{I_{s}}{I_{u}+I_{s}} \cdot \operatorname{MTF}_{S}(R, \lambda)\right],
\end{aligned}
$$

where $I_{u}$ and $I_{s}$ are the intensities of the unscattered and scattered light that enters the imaging system. The sum of $I_{u}$ and $I_{s}$ yields the total entered light intensity. Considering the boundary layer turbulence, the MTF in Eq. (7) might be introduced into $\mathrm{Eq}$. (8) as a multiplication factor.

Equation (8) yields only an approximate description of the atmospheric influences. In reality, the connection between the individual MTFs may be much more complicated, because all particular influences are dependent on each other and connected by the meteorological parameters. For example, the wavefront of a plane wave is distorted by the atmospheric turbulence and deviates from the ideal plane wave. Before entering the imaging system the distorted wavefront is additionally deformed by the aircraft boundary layer turbulence. Theoretically it is possible that the deformation of the wavefront caused by atmospheric turbulence is compensated by the boundary layer turbulence. Thereby, the resulting deviation of the wavefront from the plane wave is reduced, which leads to an image quality improvement. Furthermore, this may be valid for the connection between the atmospheric influences and aberrations of the aerial camera lens. Therefore, not only the combination of all particular atmospheric influences must be examined further but also their connection to other links of the image transfer chain.

\section{Dependence of the Refractive Index Structure Constant on Altitude}

The atmospheric turbulence is described with Fried's parameter. As shown in Eq. (2), Fried's parameter is defined by an integral over the refractive index structure constant and is dependent on altitude, temperature, and air pressure. The refractive index structure constant $C_{n}^{2}$ is a measure for the strength of the present turbulence. Using the formula obtained by Friehe et al., ${ }^{19}$ the connection of the refractive index structure constant $C_{n}^{2}$ with temperature, air pressure, and air humidity is derived to be (see Sec. 6, Appendix)

$$
\begin{aligned}
C_{n}^{2}(h)= & \left\{80 \times 10^{-6}\left[P(h) / T^{2}(h)\right]\right\}^{2} \cdot C_{t}^{2}(h) \\
& +9.0672 \times 10^{-12}\left[P(h) / T^{2}(h)\right] \cdot C_{t q}(h) \\
& +3.2115 \times 10^{-15} C_{q}^{2}(h),
\end{aligned}
$$

where

$$
P=\text { the air pressure in millibar (mb) }
$$

$$
\begin{aligned}
& T=\text { the temperature in Kelvin }(\mathrm{K}) \\
& C_{t}^{2}=\text { the temperature structure constant } \\
& C_{t q}=\text { the temperature-water vapor structure coefficient } \\
& C_{q}^{2}=\text { the humidity structure constant. }
\end{aligned}
$$

For optical propagation in dry air and in the case of most astronomical observations, the second and third term in Eq. (9) can be negligible. ${ }^{11.19-21}$ Therefore, Eq. (9) becomes

$C_{n}^{2}(h)=\left\{80 \times 10^{-6}\left[P(h) / T^{2}(h)\right]\right\}^{2} \cdot C_{t}^{2}(h)$.

All parameters in Eqs. (9) and (10) are a function of the altitude. To calculate the integral in Eq. (2) and to determine Fried's parameter for a given flight altitude, the refractive index structure constant dependency on altitude must be deduced. It has been examined to describe $C_{n}^{2}$ through the altitude $h$ only. For this reason, different models have been suggested. $9.20 .22-24$ They can be condensed by the following general formula:

$C_{n}^{2}(h)=C_{n o}^{2} h^{-b} \exp \left(-h / h^{\prime}\right)$,

where $C_{n o}^{2}, b$, and $h^{\prime}$ are the chosen parameters.

Fried's model ${ }^{9}$ :

$b=1 / 3$,

$h^{\prime}=3200 \mathrm{~m}$,

$C_{n o}^{2}=4.22 \times 10^{-14} \mathrm{~m}^{-1 / 3}$,

$C_{n}^{2}(h)=4.22 \times 10^{-14} h^{-1 / 3} \exp (-h / 3200) \quad\left(\mathrm{m}^{-2 / 3}\right)$.

\section{Brookner's model ${ }^{23}$ :}

$b=5 / 6$,

$h^{\prime}=320 \mathrm{~m}$,

$C_{m o}^{2}=3.6 \times 10^{-13} \mathrm{~m}^{1 / 6}$,

$C_{n}^{2}(h)=3.6 \times 10^{-13} h^{-5 / 6} \exp (-h / 320) \quad\left(\mathrm{m}^{-2 / 3}\right)$.

Tatarski's model ${ }^{20}$.

$b=4 / 3$,

$\boldsymbol{h}^{\prime}=\infty$,

$C_{m o}^{2}=4.16 \times 10^{-13} \mathrm{~m}^{2 / 3}$,

$C_{n}^{2}(h)=4.16 \times 10^{-13} h^{-4 / 3} \quad\left(\mathrm{~m}^{-2 / 3}\right)$.

Hufnagel's model ${ }^{24}$ :

$$
\begin{aligned}
C_{n}^{2}(h)= & 2.7 \times 10^{-16}\left[2.2 \times 10^{-37} h^{10}(w / 27)^{2}\right. \\
& \times \exp (-h / 1000)+\exp (-h / 1500)] \quad\left(\mathrm{m}^{-2 / 3}\right) .
\end{aligned}
$$

The wind is one of the most important factors for atmospheric turbulence. In Hufnagel's model, its influence is considered with a factor $w$, which is the average wind speed in the range of elevations from 5 to $20 \mathrm{~km}$. Equation (15) is actually a 


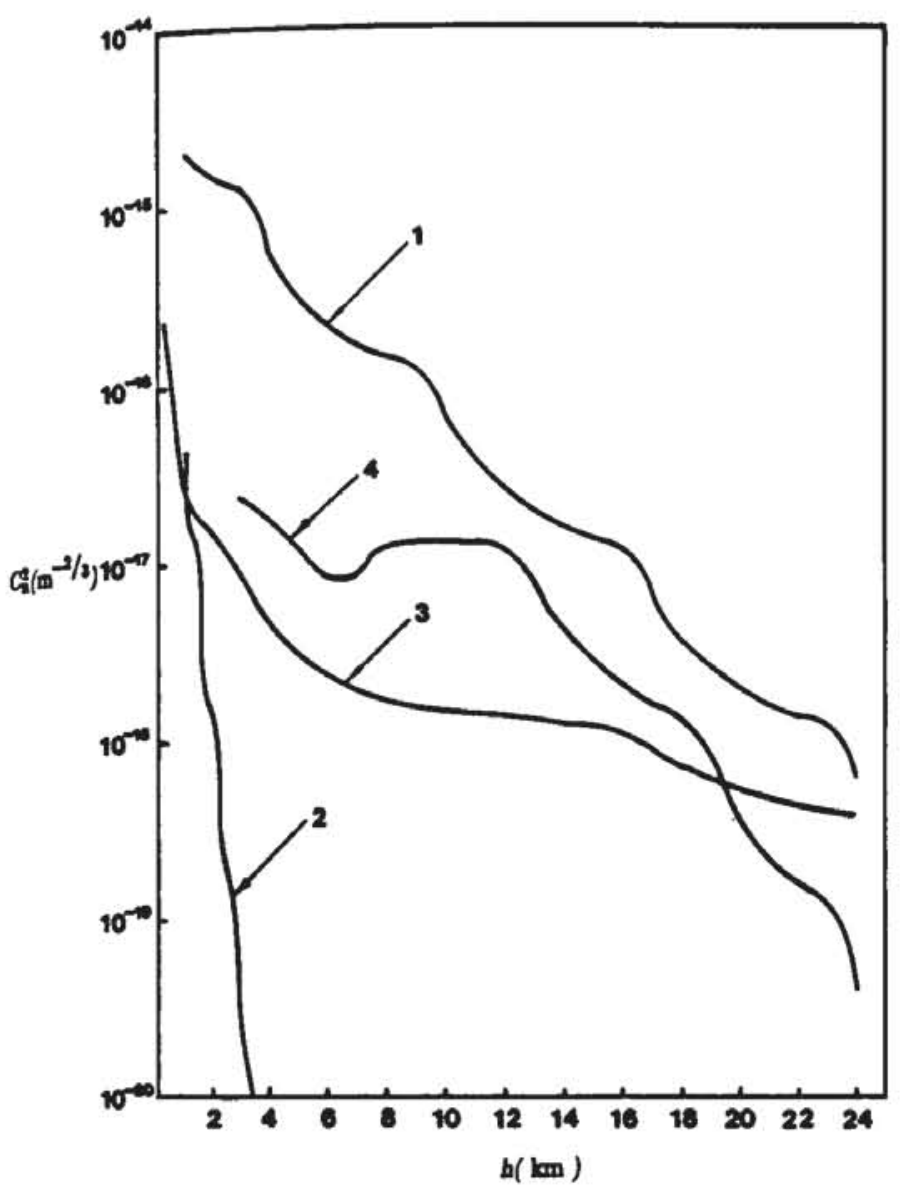

Fig. 2 Curves of the refractive index structure constant via the altitude 1, Fried's model; 2, Brookner's model; 3, Tatarski's model; and 4, Hufnagel's model. (For the Hufnagel's model, $w=18 \mathrm{~m} / \mathrm{s}$ was assumed.)

combination of two parts, each of which has the form of Eq. (11). For the first part, $b=-10, h^{\prime}=1000 \mathrm{~m}$, $C_{m o}^{2}=5.94 \times 10^{-53} \mathrm{~m}^{-32 / 3}$, and a weight factor $(w / 27)^{2}$ are put into Eq. (11). In the second part are $b=0, h^{\prime}=1500 \mathrm{~m}$, and $C_{n o}^{2}=2.7 \times 10^{-16} \mathrm{~m}^{-2 / 3}$. The model in Eq. (15) is only valid for the range of elevations from $3 \mathrm{~km}$ above the local ground level to $24 \mathrm{~km}$ above the sea level. ${ }^{24}$ The model in Eq. (14) is a theoretical model while the other models are based on experimental measurements. The models have been checked under different weather conditions and measurement fields. ${ }^{21.22 .25-29}$ Tatarski's model appears to fit best for small altitudes, ${ }^{22,29}$ while the other models are more suited to higher altitudes. ${ }^{21,25-28}$ However, the vertical $C_{n}^{2}(h)$ profiles of the atmosphere, measured by Ochs et al. ${ }^{26}$ at night, indicate increases in $C_{n}^{2}$ at altitudes on the order of 10 to $20 \mathrm{~km}$. These results contradict the models discussed in this section.

The curves of $C_{n}^{2}(h)$ for all four models are shown in Fig. 2. They vary smoothly over the propagation path. With the help of the models, the integral in Eq. (2) can be calculated for a given location and altitude, and Fried's parameter $r_{0}$ can be determined. The result of the integral calculation corresponds to the plane in Fig. 2, which is limited by the curve and the abscissa in a given altitude range. According to Eq. (2), the larger this plane is, the smaller is Fried's parameter $r_{0}$. A small Fried's parameter corresponds to strong turbulence. Curve 1 is calculated according to Fried's model and lies above all other curves in Fig. 2. Therefore, it yields the strongest turbulence among all four curves. According to the models
Table 1 Photographic data.

\begin{tabular}{|c|c|}
\hline Deser & Juby $2 a, 150$ \\
\hline Test terint: & 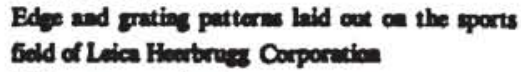 \\
\hline Altitude of the aports felds & 406 m above ses lend \\
\hline Aircmat: & Pilatus Turbo Parter PC-6 \\
\hline Camare: & RC 20 (WILD) ned with FMC \\
\hline Objectives & $30 / 4$ NAT $-F, f=303.76 \mathrm{~mm}$ \\
\hline Filur: & $\begin{array}{l}\text { Yellow fiter } 120 \mathrm{am} \text { form } \mathrm{Na}, 1,2 \text { and } 3 \\
\mathrm{Na}, 4 \text { with the filter } 525 \mathrm{em}\end{array}$ \\
\hline Flan & Kodak Panatcamio-X 2412 \\
\hline Gamosomaluse & $\sim 1.3$ \\
\hline Expoevere these: & 1/1000s \\
\hline $\begin{array}{l}\text { Secting woditions } \\
\text { near the growed }\end{array}$ & 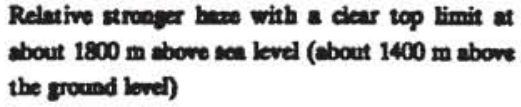 \\
\hline
\end{tabular}

\begin{tabular}{|c|c|c|c|c|}
\hline \multirow[t]{2}{*}{$\mathrm{Na}$} & \multirow{2}{*}{ (b) } & \multirow{2}{*}{$\begin{array}{l}\text { figigs altitude } \\
\text { chove sea leved }\end{array}$} & \multicolumn{2}{|c|}{ visibility from the aircrat } \\
\hline & & & inclioed sight & borizontal sight \\
\hline 1 & 12.06 & $900 \mathrm{~m}$ & $12-18 \mathrm{~km}$ & $27 \mathrm{~km}$ \\
\hline 2 & 1216 & $1920 \mathrm{~m}$ & $30-45 \mathrm{~km}$ & $40-50 \mathrm{~km}$ \\
\hline 3 & 12.35 & $3435 \mathrm{~m}$ & $30-50 \mathrm{~km}$ & $90-150 \mathrm{~km}$ \\
\hline 4 & 12.43 & $3035 \mathrm{~m}$ & & \\
\hline
\end{tabular}

\begin{tabular}{|c|c|c|c|c|}
\hline \multicolumn{5}{|c|}{ Mensurements on the ground near the test pattens } \\
\hline $\mathrm{Na}$ & temper- & $\begin{array}{l}\text { relative } \\
\text { bumidity }\end{array}$ & $\begin{array}{l}\text { air preature } \\
\text { (mb) }\end{array}$ & $\begin{array}{l}\text { borinoutal } \\
\text { illuminarioge }\end{array}$ \\
\hline 1 & $2{ }^{\circ} \mathrm{C}$ & $58 \%$ & $9 \pi$ & $72000 \mathrm{Lax}$ \\
\hline 2 & $205{ }^{\circ} \mathrm{C}$ & $57 \%$ & 97 & $77^{\circ} 000 \mathrm{Lax}$ \\
\hline 3 & $23{ }^{\circ} \mathrm{C}$ & $34 \%$ & 97 & $76000 \mathrm{Lax}$ \\
\hline 4 & & & & $76000 \mathrm{Lax}$ \\
\hline
\end{tabular}

mentioned above, the influence of the atmospheric turbulence increases until a certain flight altitude is reached, which is dependent on the used model. As shown in Fig. 2, the fluctuations of refractive index become very small over the model-dependent altitude so that it may be neglected. Therefore, the effect of the atmospheric turbulence will be approximately constant when the altitude exceeds a certain value.

\section{Measured Results for Different Flight Altitudes}

For the measurements, two artificial edge and grating patterns are laid out on the ground perpendicular to each other as shown in Fig. 1. To examine the atmosphere, four airborne photographs were taken by Leica Heerbrugg Corporation, Heerbrugg, Switzerland, from three different flight altitudes. For a flight altitude of about $3400 \mathrm{~m}$ above sea level, two successive photographs were taken. The weather condition and camera parameters are summarized in Table 1. During the photography, there was a relatively strong haze with a clear top limit at about $1800 \mathrm{~m}$ above sea level. As shown in Table 1, these four airborne photographs were taken shortly after each other, so that the same atmospheric conditions could be assumed. The photographs were evaluated by the edge gradient analysis (EGA) ${ }^{30}$ and the grating pattern method, ${ }^{2}$ respectively. The entire MTFs for different flight altitudes were determined not only with artificial edge patterns but also with grating patterns. Figure 3 shows only the MTF curves measured from edge images. The same results have been obtained with grating patterns. 

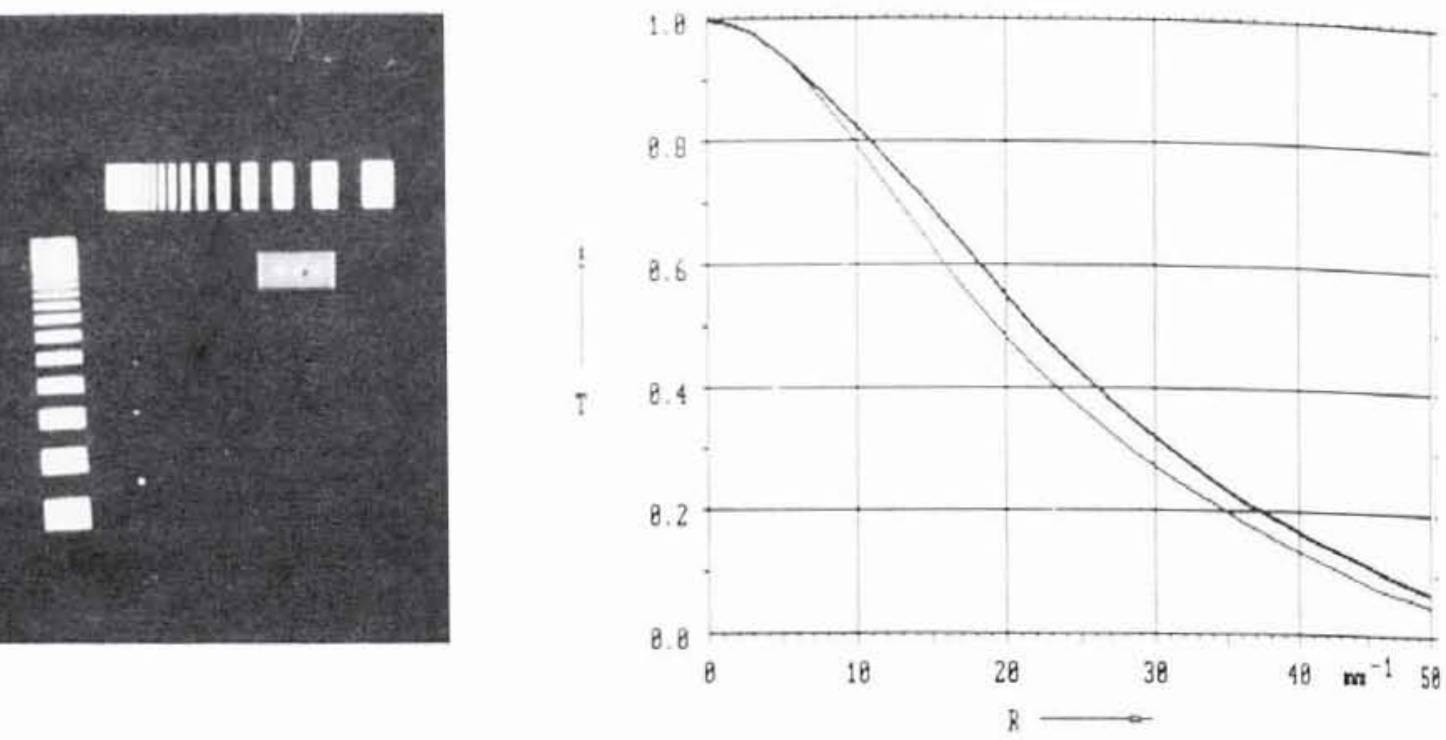

(a)
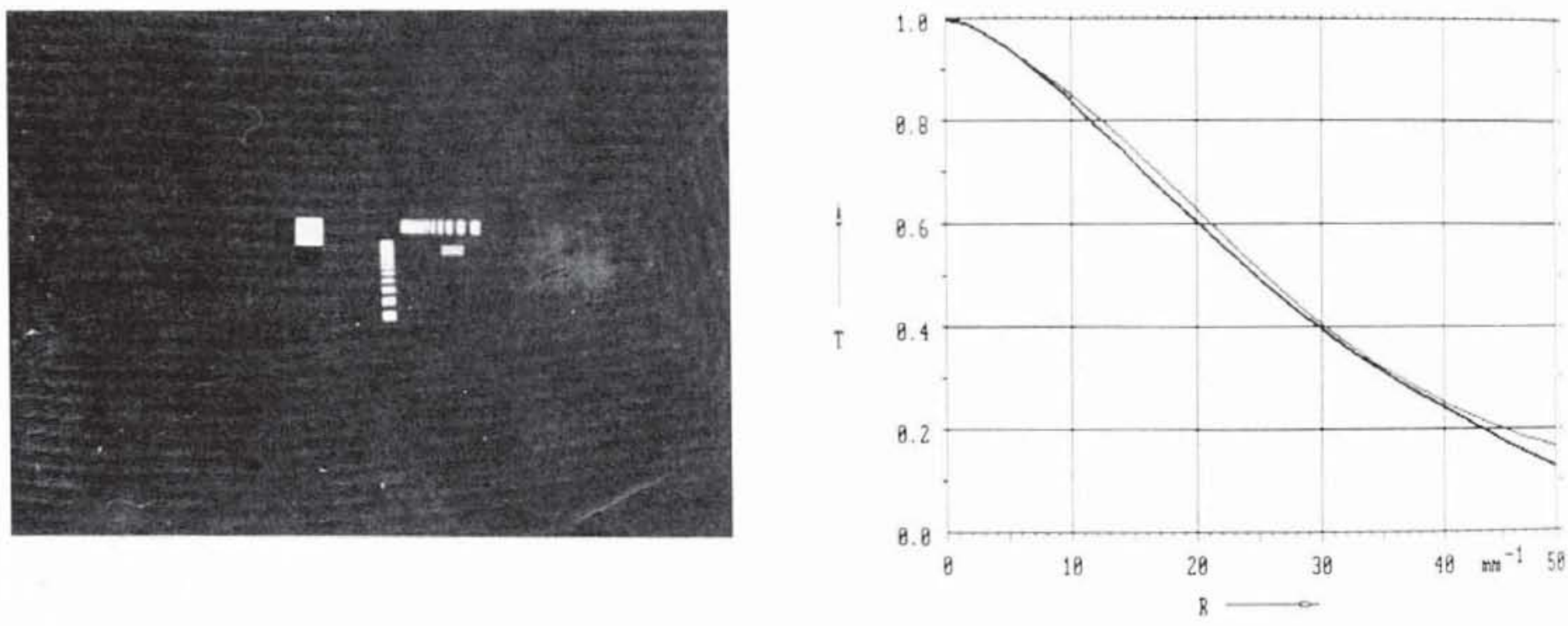

(b)
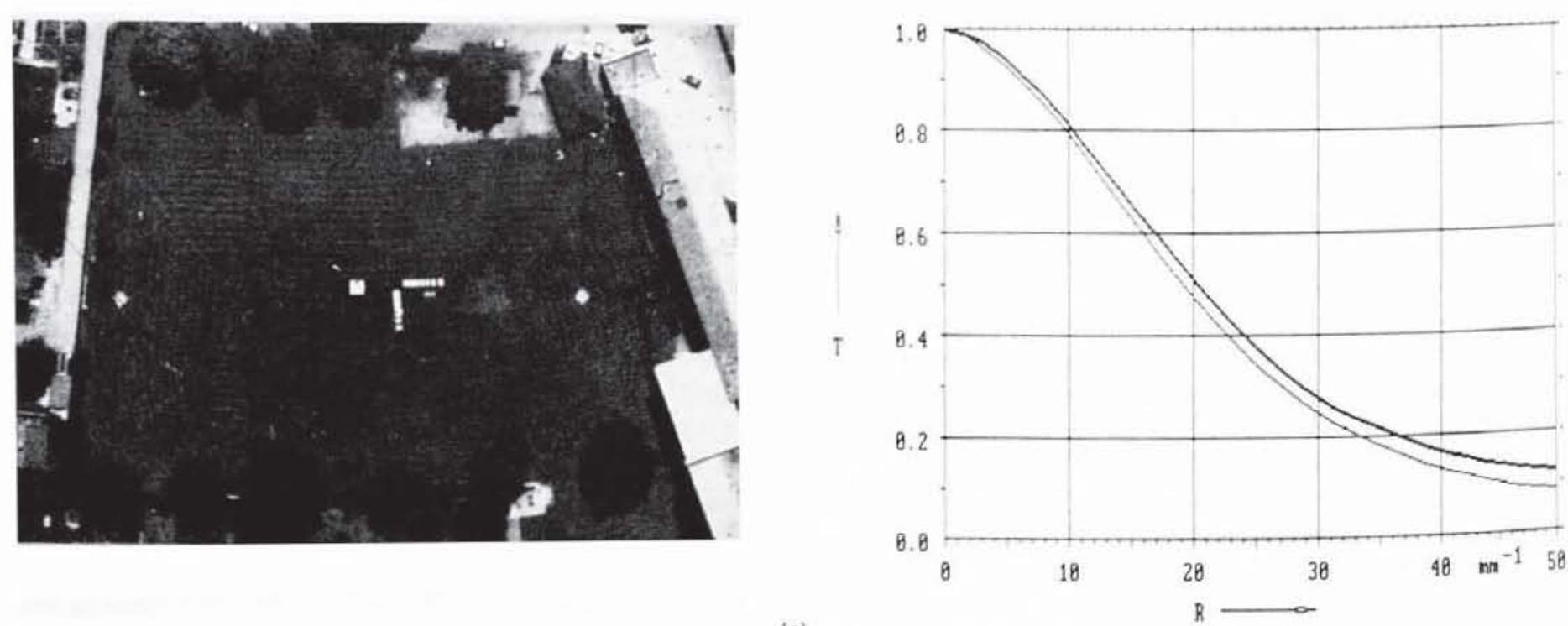

(c)

Fig. 3 Airborne photographs taken at three different flight altitudes and the corresponding MTF curves: W. MTF obtained with the edge image in $X$ direction and. MTF obtained with the edge image in $Y$ direction: (a) $H=900 \mathrm{~m}$ above sea level, (b) $H=1920 \mathrm{~m}$ above sea level, and (c) $H=3435 \mathrm{~m}$ above sea level. 
In Fig. 3, two MTF curves for each flight altitude are shown together. The curves in the $X$ direction correspond to the patterns in flight direction, and the $Y$ direction is perpendicular to it. We found good agreement of the MTF curves obtained from both edge patterns. Because of the larger transfer path, the MTF for a flight altitude of $3435 \mathrm{~m}$ above sea level is worse than the one for $1920 \mathrm{~m}$ above sea level. According to Eqs. (2), (3), and (5), the MTF of different atmospheric influences decreases with altitude. But unexpectedly, the curves for a flight altitude of $900 \mathrm{~m}$ above sea level lie lower than those for $1920 \mathrm{~m}$ above sea level. This is probably due to the relative strong haze below $1800 \mathrm{~m}$ above sea level. At $900 \mathrm{~m}$, the haze was close to the entrance pupil of the camera, which lead to a considerable influence on the image quality - the nearer the turbulent layer to the camera entrance pupil, the greater the influence on image quality is expected. On the other hand, the values of $C_{n}^{2}$ near the image make a great contribution to the influence of the atmospheric turbulence. At the altitudes of the haze layer, $C_{n}^{2}$ can be higher, thus the turbulence near the image will be stronger. Although this supposition does not agree with the models of $C_{n}^{2}$, there are some measurements of $C_{n}^{2}$ height profiles with increases in $C_{n}^{2}$ at a range of altitude ${ }^{26}$ as mentioned in the previous section. A systematic study of the specific effects of weather parameters on $C_{n}^{2}$ indicates that $C_{n}^{2}$ increases with aerosol concentration and size. ${ }^{31}$ Therefore, the strong haze appears to be associated with a $C_{n}^{2}$ increase at the lowest altitude. Furthermore, it is possible that the aircraft has created a stronger boundary layer turbulence due to the strong haze. Moreover, the visibility from the aircraft was the worst at $900 \mathrm{~m}$ (see Table 1). For example, the inclined sight visibility was 12 to $18 \mathrm{~km}$. To image an object on the ground, the inclined sight visibility is essential. The bad visibility reduces the contrast of the object and the image. At 1920 and $3435 \mathrm{~m}$, the inclined sight visibility was approximately equal.

For a flight altitude of $3435 \mathrm{~m}$, we examined two photographs taken one after the other in a time interval of $8 \mathrm{~min}$. Figure 4 shows the MTF curves measured on both photographs. The curves obtained from the edge pattern in one direction are shown in Fig. 4(a) while the ones obtained in a perpendicular direction are shown in Fig. 4(b). They are in good agreement although a filter was changed between the photographs. It can be shown that the atmosphere affected both photographs taken under equal conditions equally.

There are two methods to check the models of the atmospheric influences. If the MTF curves of the camera lens and film are known, the atmospheric MTF can be experimentally determined by dividing the measured MTF by the known MTFs. It can be compared with the theoretical models. On the other hand, the models can be checked by dividing the measured MTF by the theoretical atmospheric MTF calculated according to the chosen model. If all MTFs obtained after division agree for different flight altitudes, the used model could be considered experimentally verified on the supposition that all other factors remain unchanged.

A comparison between measured and calculated MTF curves is made at two different flight altitudes. If the MTF at altitude $h_{1}$ is denoted with $\operatorname{MTF}\left(h_{1}\right)$, and at $h_{2}$ with $\operatorname{MTF}\left(h_{2}\right)$, where $h_{2}$ is greater than $h_{1}$, we obtain

$\frac{\operatorname{MTF}_{\text {res }}\left(h_{2}\right)}{\operatorname{MTF}_{\text {res }}\left(h_{1}\right)}=\frac{\operatorname{MTF}_{A}\left(h_{2}\right)}{\operatorname{MTF}_{A}\left(h_{1}\right)}$.

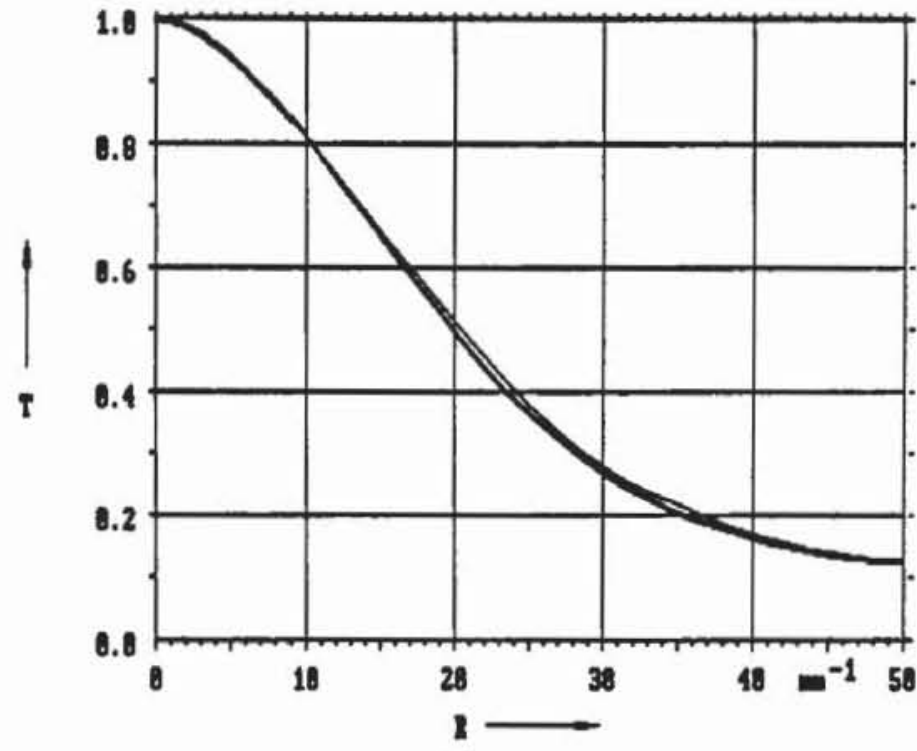

(a)

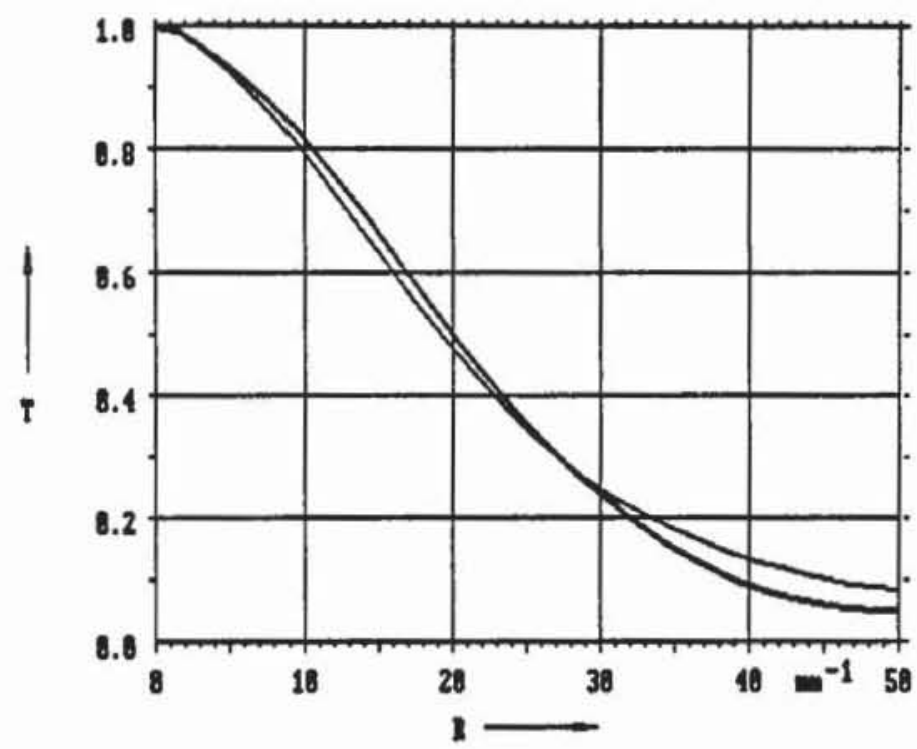

(b)

Fig. 4 MTF curves measured from two airbome photographs taken with the same altitude ( $H=3435 \mathrm{~m}$ above sea level): (a) MTF in $X$ direction and (b) MTF in $Y$ direction.

where $\operatorname{MTF}_{\text {res }}\left(h_{2}\right)$ and $\operatorname{MTF}_{\text {res }}\left(h_{1}\right)$ are the measured resulting MTF and $\operatorname{MTF}_{A}\left(h_{2}\right)$ and $\operatorname{MTF}_{A}\left(h_{1}\right)$ are the atmospheric MTFs at the altitudes $h_{2}$ and $h_{1}$, respectively. We assume that other influences, such as vibrations, are constant at both flight altitudes. From Eq. (16), it follows that

$\operatorname{MTF}_{\text {res }}\left(h_{2}\right)=\frac{\operatorname{MTF}_{A}\left(h_{2}\right)}{\operatorname{MTF}_{A}\left(h_{1}\right)} \cdot \operatorname{MTF}_{\text {res }}\left(h_{1}\right)$.

To verify the model, the MTF at $h_{2}$, calculated by Eq. (17), can be compared with the measured MTF obtained at $h_{2}$. To estimate the atmospheric MTF, only atmospheric turbulence is considered at first. Fried's model, which describes a relatively strong turbulence, has been used as an example. Figure 5 shows MTF curves calculated with Fried's parameters for different flight altitudes. The ratio of $\operatorname{MTF}_{A}(3435)$ to $\mathrm{MTF}_{A}(1920)$ is shown in Fig. 6. If this ratio and the measured MTF for $1920 \mathrm{~m}$ [corresponding to the fat curve in Fig. 3(b)] 


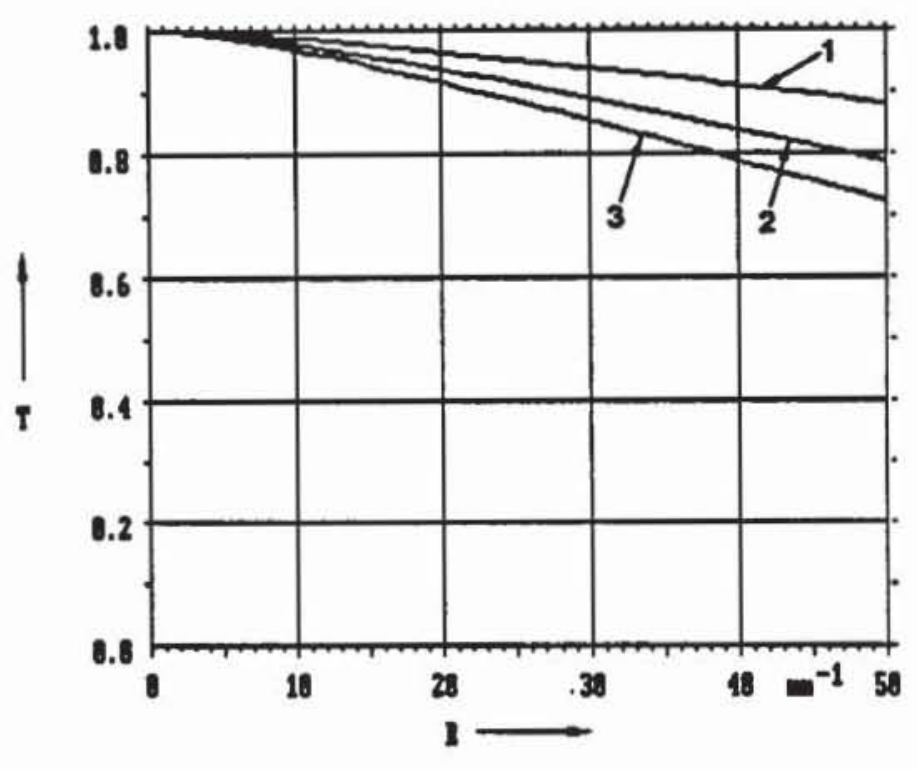

Fig. 5 MTF of the atmospheric turbulence calculated according to the Fried's model: $1, H=900 \mathrm{~m}$ above sea level, $r_{0}=40.95 \mathrm{~mm} ; 2$, $H=1920 \mathrm{~m}$ above sea level, $r_{0}=28.09 \mathrm{~mm}$; and $3, H=3435 \mathrm{~m}$ above sea level, $r_{0}=23.44 \mathrm{~mm}$.

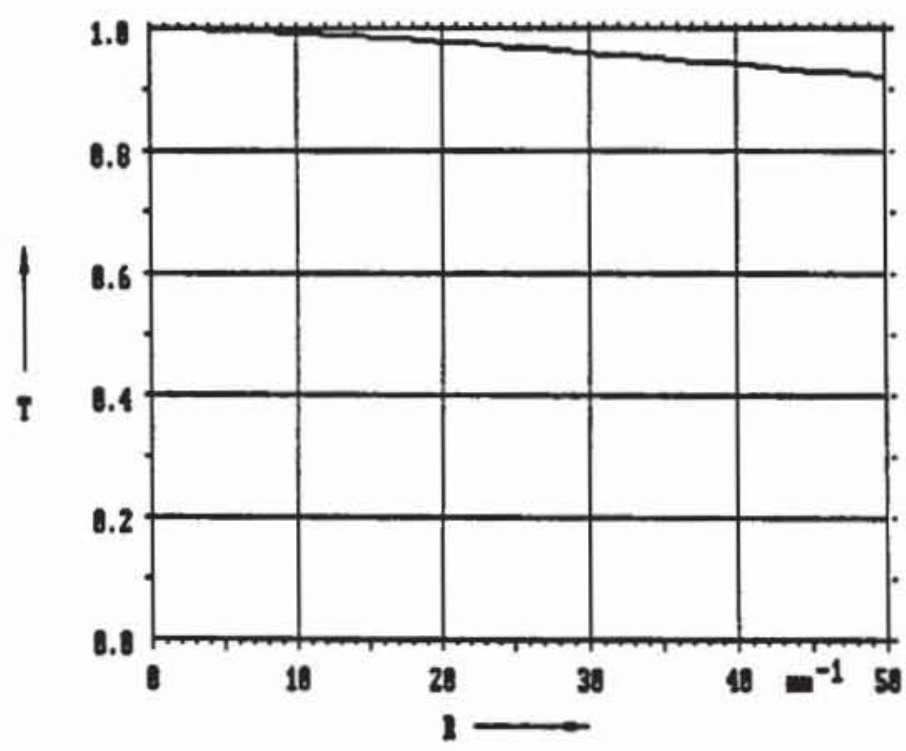

Flg. 6 Relative MTF of turbulence between the altitudes 1920 and $3435 \mathrm{~m}$.

are put into Eq. (17), the calculated MTF for the flight altitude $3435 \mathrm{~m}$ results. It corresponds to curve 3 in Fig. 7 .

Figures 6 and 7 show that the influence of the atmospheric turbulence according to Fried's model should be small. The difference between curves 1 and 2 in Fig. 7 indicates that the influence of atmospheric turbulence is greater than predicted by the theoretical model. An explanation could be that additional atmospheric influences, such as those mentioned in Sec. 2, need to be taken into consideration. It is unlikely that mechanical influences from the camera carrier were different for our photographs. This can be supported by the good agreement of the curves in Fig. 4. The aircraft boundary layer turbulence, however, might be different at different photographs. According to the theory, the contrast degradation due to atmospheric background and the forward scattering by airborne particles are dependent on the altitude, in which the

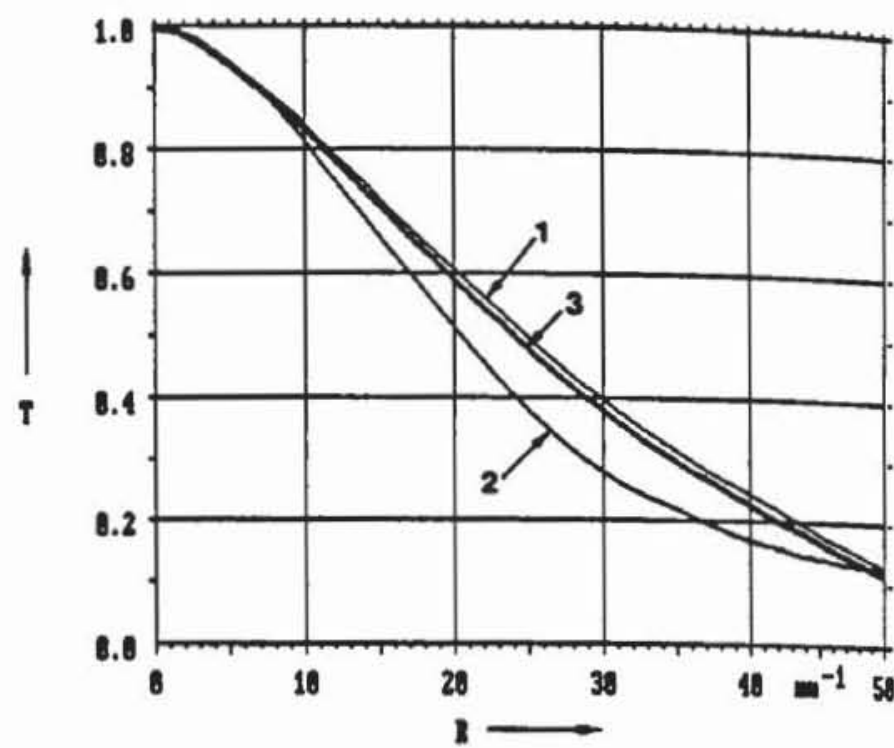

Flg. 7 MTF measured for flight altitude (curve 1) $1920 \mathrm{~m}$ above sea level and (curve 2) $3435 \mathrm{~m}$ above sea level, and (curve 3) MTF calculated by multiplying curve 1 with the relative MTF of the turbulence.

former is independent on the spatial frequency, and the latter quasi-independent on it. However, both influences appear to increase with increasing spatial frequencies according to the measurements. Therefore, it is necessary to enlarge and modify the models.

\section{Conclusions}

The MTF of the atmosphere was examined. The measurements show that the atmosphere can be a limiting link in the image-forming chain. According to a comparison between measurements and calculations, it seems that it is not enough to consider the atmospheric turbulence as a disturbing factor only. Additional disturbing factors in the atmosphere need to be considered. The atmospheric influences are dependent on the flight altitude. This dependence is comprehended in the different models and checked through the measurement of airborne photographs taken at different flight altitudes. According to the models, the atmospheric influences increase with flight altitude, but depending on the weather conditions, this can change, especially if haze is close to the entrance pupil of the camera. This is verified by some measurements. The combination of the MTFs of different atmospheric influences must be examined further.

\section{Appendix}

In Ref. 19, it has been indicated that fluctuations of refractive index are shown to be composed of fluctuations of the temperature, pressure, and water vapor content of air for the visible spectrum. A corresponding equation for the fluctuating refractivity has been given by Friehe et al. ${ }^{19}$ as

$n^{\prime}=-1.00 \theta+0.366 p-0.05667 q$,

where

$n^{\prime}=$ the fluctuation of the refractivity

$\theta=$ the fluctuation of the temperature $\left({ }^{\circ} \mathrm{C}\right)$

$p=$ the fluctuation of the pressure (millibars)

$q=$ the fluctuation of the absolute humidity $\left(\mu \mathrm{g} / \mathrm{cm}^{3}\right)$. 
The second term in Eq. (18) can be neglected due to the small contribution of the fluctuating static pressure field. Therefore, Eq. (18) is reduced to

$n^{\prime}=-1.00 \theta-0.05667 q$.

It should be noted that the coefficient to $\theta$ is calculated under the mean conditions of $\bar{T}=15^{\circ} \mathrm{C}$ and $P_{0}=760$ torr, whereas the coefficient of the absolute humidity does not depend on the mean pressure $P_{0}$ or temperature $\bar{T}$ of the air. ${ }^{19}$ To universalize Eq. (19), we use the formula obtained by Tatarski ${ }^{20}$ instead of the coefficient of -1.00 . Therefore, Eq. (19) becomes

$n^{\prime}=-80 \cdot \frac{P_{m}}{\bar{T}_{a}^{2}} \cdot \theta-0.05667 q$,

where $P_{m}$ is the mean pressure in millibar and $\bar{T}_{a}$ is the mean temperature in degrees Kelvin. There is a small difference between the formula of Tatarski and the formula of Friehe, for example, using the formula of Tatarski with $\bar{T}_{a}=288.16 \mathrm{~K}$ $\left(15^{\circ} \mathrm{C}\right)$ and $P_{m}=1010.8 \mathrm{mb}$ (760 torr), the coefficient of $\theta$ is 0.974 .

It should be remembered that $n^{\prime}=\mathrm{d} N$ is denoted by Friehe, ${ }^{19}$ where $N=(n-1) \times 10^{6}$ is the refractivity $(n$ is the refractive index). Therefore, we have

$n^{\prime}=\mathrm{d} N=\mathrm{d} n \times 10^{6}$.

We denote $\mathrm{d} n$ by $n$ ". Substituting the expression (21) into Eq. (20), we obtain the fluctuation of the refractive index as

$n^{n}=-80 \times 10^{-6} \cdot \frac{P_{m}}{\bar{T}_{a}^{2}} \cdot \theta-5.667 \times 10^{-8} q$.

From Eq. (22), the variance of $n^{n}$ is given by

$$
\begin{aligned}
\overline{n^{n 2}}= & \left(80 \times 10^{-6} \cdot \frac{P_{m}}{\bar{T}_{a}^{2}}\right)^{2} \cdot \overline{\theta^{2}}+9.0672 \times 10^{-12} \\
& \times \frac{P_{m}}{\bar{T}_{a}^{2}} \cdot \overline{\theta q}+3.2115 \times 10^{-15} \bar{q}^{2} ;
\end{aligned}
$$

and the power spectrum of $n^{n}$ is

$$
\begin{aligned}
\phi_{n^{*}}(f)= & \left(80 \times 10^{-6} \cdot \frac{P_{m}}{\bar{T}_{a}^{2}}\right)^{2} \cdot \phi_{\theta}(f) \\
& +9.0672 \times 10^{-12} \cdot \frac{P_{m}}{\bar{T}_{a}^{2}} \cdot C o_{\theta q}(f) \\
& +3.2115 \times 10^{-15} \phi_{q}(f),
\end{aligned}
$$

where

$$
\begin{aligned}
& \overline{n^{\overline{2}}}=\int_{0}^{\infty} \phi_{n^{-}}(f) \mathrm{d} f, \\
& \overline{\theta^{2}}=\int_{0}^{\infty} \phi_{\theta}(f) \mathrm{d} f,
\end{aligned}
$$

$$
\begin{aligned}
& \overline{\theta q}=\int_{0}^{\infty} C o_{\theta q}(f) \mathrm{d} f, \\
& \overline{q^{2}}=\int_{0}^{\infty} \phi_{q}(f) \mathrm{d} f,
\end{aligned}
$$

and $f$ is the frequency in hertz.

According to early studies, ${ }^{11.19 .20}$ the power spectra of $n^{n}$, $\theta, q$, and the cospectrum term of $\theta q$ should be described by

$$
\begin{aligned}
& \phi_{n^{-}}(f)=A C_{n}^{2} f^{-5 / 3} \\
& \phi_{\theta}(f)=A C_{t}^{2} f^{-5 / 3} \\
& C o_{\theta q}(f)=A C_{t q} f^{-5 / 3} \\
& \phi_{q}(f)=A C_{q}^{2} f^{-5 / 3},
\end{aligned}
$$

where $C_{n}^{2}$ is the refractive index structure constant, $C_{t}^{2}$ is the temperature structure constant, $C_{t q}$ is the temperature water vapor structure coefficient, and $C_{q}^{2}$ is the humidity structure constant; $A$ is the same parameter for all equations. Putting Eqs. (25) through (28) into Eq. (24), we obtain

$$
\begin{aligned}
C_{n}^{2}= & \left(80 \times 10^{-6} \cdot \frac{P_{m}}{\bar{T}_{a}^{2}}\right)^{2} \cdot C_{t}^{2}+9.0672 \times 10^{-12} \\
& \times \frac{P_{m}}{\bar{T}_{a}^{2}} \cdot C_{t q}+3.2115 \times 10^{-15} C_{q}^{2} .
\end{aligned}
$$

Because of the dependence of $P_{m}$ and $\bar{T}_{a}$ on altitude, we denote $P_{m}$ and $\bar{T}_{a}$ with $P(h)$ and $T(h)$, respectively. Therefore, the refractive index structure constant is given by

$$
\begin{aligned}
C_{n}^{2}(h)= & \left\{80 \times 10^{-6}\left[P(h) / T^{2}(h)\right]\right\}^{2} \cdot C_{t}^{2}(h) \\
& +9.0672 \times 10^{-12}\left[P(h) / T^{2}(h)\right] \cdot C_{t q}(h) \\
& +3.2115 \times 10^{-15} C_{q}^{2}(h) .
\end{aligned}
$$

\section{Acknowledgments}

We would like to thank Leica Heerbrugg Corporation for the submission of the airborne photographs referred to. We would like to express sincere gratitude to the referees for their comments on the earlier version of this paper.

\section{References}

1. H.-K. Meier, "Progress by forward motion compensation for Zeiss aerial cameras," Bildmessung und Lufibildwesen 52, 143-152 (1984).

2. F. Lei and H. J. Tiziani, "A comparison of methods to measure the modulation transfer function of aerial survey lens systems from the image structures," Photogram. Eng. Remote Sensing 54(1), 41-46 (1988).

3. T. Trott, "The effects of motion on resolution," Photogramm. Eng. 26, $819-827$ (1960).

4. D. Wulich and N. S. Kopeika, "Image resolution limits resulting from mechanical vibrations," Opt. Eng. 26, 529-533 (1987).

5. H. Tiziani, "Beurteilung der Bildqualităt von Luftbildkammem," Vermessung, Photogrammetrie, Kulturtechnik, pp. 4-77 and 137-146 (1977). 
6. N. S. Kopeika, "Imaging through the atmosphere for airborne reconnaissance," Opt. Eng. 26(11), 1146-1154 (1987).

7. E. W. Smith, "Optical effects of aircraft boundary layer turbulence," Appl. Opt. 26(3), 529-532 (1987).

8. A. Chelli, P. Lena, C. Roddier, F. Roddier, and F. Sibille, "Modulation transfer function for infra-red stellar speckle interferometry: evidence for a log-normal statistic," Optica Acta 26(5), 583-595 (1979).

9. D. L. Fried, "Statistics of a geometric representation of wavefront distortion," J. Opt. Soc. Am. 55(11), 1427-1435 (1965).

10. D. L. Fried, "Optical resolution through a randomly inhomogeneous medium for very long and very short exposures," J. Opt. Soc. Am. 56(10), 1372-1379 (1966).

11. F. Roddier, "The effects of atmospheric turbulence in optical astronomy," in Progress in Optics XIX, E. Wolf, Ed., pp. 281-376. NorthHolland (1981).

12. D. L. Walters, D. L. Favier, and J. R. Hines, "Vertical path atmospheric MTF measurements," J. Opt. Soc. Am. 69(6), 828-837 (1979).

13. D. L. Fried and G. E. Mevers, "Evaluation of $r_{0}$ for propagation down through the atmosphere," Appl. Opt. 13(11), 2620-2622 (1974).

14. J. W. Hardy, "The role of active optics in large telescopes," in E.S.O. Conf, on Optical Telescopes of the Future, Geneva, F. Pacini, W. Richter, and R. N. Wilson, Eds., pp. 455-467 (1978).

15. J. L. Horner. "Optical restoration of images blurred by atmospheric turbulence using optimum filter theory," Appl. Opt. 9(1), 167-171 (1970).

16. M. Michaelis, E. Dennert-Moller, and M. Ehlers, "Zur Korrektur radiometrischer Störeinflüsse in Fernerkundungsbildern mit Hilfe statistischer Verfahren," Bildmessung und Luffibildwesen 53, 163-173 (1985).

17. R. Gru $\beta$, "Ubertragung von Laserstrahlung durch die Atmosphäre," Nachr.-Techn. Z 22, 184-192 (1969),

18. D. Kelsall and R. D'Amato, "AFWL/OPTICS optical degradation by aerodynamic boundary layers, " in MIT-Lincoln Laboratory Repont ESD-TR-78-243 (1977).

19. C. A. Friehe, J. C. La Rue, F. H. Champagne, C. H. Gibson, and G. F. Dreyer, "Effects of temperature and humidity fluctuations on the optical refractive index in the marine boundary layer," J. Opt. Soc, Am, 65(12), 1502-1511 (1975).

20. V.I. Tatarski, Wave Propagation in a Turbulent Medium. McGrawHill, New York (1961).

21. R. Barletti, G. Ceppatelli, L. Paterno, A. Righini, and N. Speroni, " Mean vertical profile of atmospheric turbulence relevant for astronomical seeing," J. Opt. Soc. Am. 66(12), 1380-1383 (1976).

22. N. Ben-Yosef, E. Tirosh, A. Weitz, and E. Pinsky, "Refractive-index structure constant dependence on height," J. Opt. Soc. Am. 69(11). 1616-1618 (1979).

23. E. Brookner, "Improved model for the structure constant variations with altitude," Appl. Opt. 10(8), 1960-1962 (1971).

24. R. E. Hufnagel, "Variations of atmospheric turbulence," in Digest of Technical Papers, Topical Meeting on Optical Propagation through Turbulence, Optical Society of America, Washington, DC, Wal-1Wal-4 (1974).

25. J. L. Bufton, "Comparison of vertical profile turbulence structure with stellar observations," Appl. Opt. 12(8), 1785-1793 (1973).

26. G. R. Ochs, T. Wang, R. S. Lawrence, and S. F. Clifford, "Refractiveturbulence profiles measured by one-dimensional spatial filtering of scintillations," Appl. Opt. 15(10), 2504-2510 (1976).
27. T. E. VanZandt, J. L. Green, K. S. Gage, and W. L. Clark, "Vertical profiles of refractivity turbulence structure constant: Comparison of observations by the Sunset Radar with a new theoretical model," Radio Sci. 13(5), 819-829 (1978).

28. J. Vernin, R. Barletti, G. Ceppatelli, L. Paterno, A. Righini, and N Speroni, "Optical remote sensing of atmospheric turbulence: a comparison with simultaneous thermal measurements, "'Appl. Opt. 18(2), 243-247 (1979)

29. J. C. Wyngaard, Y. Izumi, and S. A. Collins, Jr., "Behavior of the refractive index-structure parameter near the ground," J. Opt. Soc. Am 61(12), 1646-1650 (1971)

30. F. Lei and H. J. Tiziani, "Eine neue Methode zur Bestimmung der MTF aus dem Kantenbild," Optik 74, 17-21 (1986)

31. Private communication.

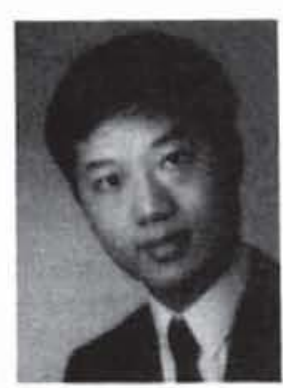

Fang Lei received the BS degree in mechanical engineering from the Huazhong University of Science and Technology, China, in 1982, and his Dr-Ing degree from the University of Stuttgart, Germany, in 1990. In October 1990 he joined the Thomson Consumer Electronics R\&D Lab. oratories in Villingen-Schwenningen, Germany, where he is working on the development of optical memory systems.

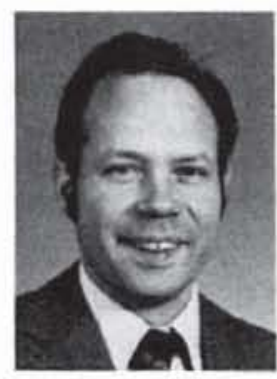

H. J. Tiziani was trained as a technician in optics and precision mechanics at Wild Heerbrugg Corporation, Heerbrugg. Switzerland (now Leica Heerbrugg Corporation) and studied mechanical engineering in St. Gallen and optics at the Institut d'Optique in Paris. After obtaining his PhD at the $1 \mathrm{~m}$ perial College, London, he directed a summer school at IBM in San Jose, California, and was a consultant to IBM. From 1968 to 1973 he developed the optics group at the Federal Institute of Technology in Zürich, Switzerland. From 1973 to 1978 he was the director of the central laboratory at Wild Heerbrugg Corporation and since 1978 he has been a director of the Institute of Applied Optics and professor at the University of Stuttgart, Germany. He was dean and deputy dean of the faculty of mechanical engineering of the University of Stuttgart and is now president of the Great Senate. In addition, he was the president of European Electro-Optics, the president of the Optics Division of the European Physical Society, and a governor of SPIE. Tiziani has written nearly 100 papers in different fields of applied optics. 